\title{
Altered Cardiovascular Control in Preterm Infants with Bronchopulmonary Dysplasia
}

\author{
SUVI VISKARI, STURE ANDERSSON, TIMO HYTINANTTI, AND TURKKA KIRJAVAINEN \\ Hospital for Children and Adolescents, University of Helsinki, 00029 HUS, Finland
}

\begin{abstract}
Vestibulo-mediated cardiovascular control in hazardous situations is important. Our hypothesis is that the prerequisite for sudden infant death syndrome (SIDS) is impaired vestibulo-mediated cardiovascular control. Prematurity is a risk factor for SIDS, and postnatal intermittent hypoxia may contribute to this risk. We studied heart rate (HR) and blood pressure (BP) responses in 10 infants with bronchopulmonary dysplasia (BPD) who were born at $27 \pm 2.4$ (23-30) wk of gestation. Twenty healthy term infants served as controls. Cardiovascular tests were performed under polysomnographic control during slow-wave sleep (SWS) at a corrected age of $12 \pm 3.5$ (7-19) wk. Control infants showed biphasic HR and BP responses to side motion with an immediate increase followed by a modest decrease and return to baseline. Compared with the controls, half of the BPD infants had altered BP responses $(p<0.005)$ without an early increase, followed by a more prominent decrease in BP. $\mathrm{BPD}$ infants also presented with a greater variability in BP responses to head-up tilts than did the controls $(p<0.001)$. In conclusion, these findings suggest that some BPD infants have impaired vestibular sympathoreflex-mediated cardiovascular control. This dysfunction may become critical in life-threatening situations. (Pediatr Res 61: 594-599, 2007)
\end{abstract}

$\mathrm{V}_{\mathrm{t}}^{\mathrm{e}}$ estibular and cerebellar dysfunction has been suggested to be part of the pathophysiology of SIDS (1). Cardiovascular control mediated by vestibular nuclei and the cerebellum are important in hazardous situations such as hypovolemic and endotoxin shock $(2,3)$. More than $80 \%$ of SIDS victims have shown neuronal apoptosis in vestibular nuclei and nuclei of the tractus solitarii, which are involved in baroand vestibular sympathoreflexes (4). SIDS victims also present with abnormalities elsewhere in the vestibular sympathoreflex pathway (5-7).

During normal daily living, vestibular sympathoreflex dysfunction is not expected to have a profound effect on cardiovascular control because several compensatory circuits are involved in control of HR and BP $(8,9)$. The dysfunction results in greater variability in BP control during postural changes (10). Similarly, increased BP variability has been observed in tilt tests performed in infants who have suffered from apparent life-threatening events (ALTEs) $(11,12)$,

Received September 6, 2006; accepted January 5, 2007.

Correspondence: Turkka Kirjavainen, M.D., Ph.D., Hospital for Children and Adolescents, P.O. Box 281, 00029 HUS, Finland; e-mail: turkka.kirjavainen@hus.fi

This study was supported by grant number TYH3230 from Helsinki University Hospital, the Sigrid Jusélius Foundation, Finska Läkaresällskapet, and the Foundation for Pediatric Research.

DOI: $10.1203 / p d r .0 b 013 e 3180459 f 43$ further supporting the idea of vestibular dysfunction in SIDS.

Most SIDS victims have suffered from hypoxia for at least several hours before the lethal event (13). SIDS victims may also have suffered from hypoxia earlier in life for multiple reasons, including placental insufficiency (14), intrauterine nicotine exposure (15), low or very low birth weight $(16,17)$, hypoxia related to obstructive apnea events $(18,19)$, or unfavorable head position during sleep $(20,21)$. Our hypothesis is that hypoxic episodes cause a dysfunction in vestibulo-mediated cardiovascular control, thus making the infant more liable to die in life-threatening situations. To test the possible effects of hypoxia and test our hypothesis, we previously studied infants with univentricular heart who present with chronic hypoxia (22). All these infants had responses to side motion that were nearly absent, indicating severe dysfunction of vestibulo-mediated cardiovascular control.

Premature infants who develop BPD commonly suffer from intermittent hypoxia during early neonatal period. They also are at increased risk of SIDS $(17,23)$. The aim of this study was to examine cardiovascular control in BPD infants. For this purpose, side-motion and tilt tests were performed, and HR variability in quiet nonrapid eye movement (NREM) sleep, and HR reactions to spontaneous arousals were analyzed in 10 preterm infants with BPD at 2-4 mo of corrected age.

\section{METHODS}

Subjects. Ten infants born at $27 \pm 2.4$ (23-30) wk and 20 full-term, age-matched control infants were studied at corrected ages of $11.4 \pm 4.0$ (7-19) and $12.1 \pm 3.3(8-19) \mathrm{wk}(p=0.66)$, respectively. All preterm infants had BPD (24) and had suffered from intermittent hypoxia despite intensive critical care and ventilatory support (Fig. 1). Demographic data of BPD infants are presented in Table 1. The controls were healthy, with an uneventful neonatal history and normal clinical examination, and their growth was within normal limits (25). The results for 11 of the 19 controls were published previously (22).

Written parental consent was obtained for all subjects. The study protocol was approved by the Ethics Review Committee of the Hospital for Children and Adolescents, Helsinki University Hospital, Helsinki, Finland.

Study design. The study methods were previously described (22). All tests were performed at night (2000-0005 h) while infants were lying supine on a mat with a rigid frame placed on the top of their usual cot mattress. Two to

Abbreviations: BP, blood pressure; BPD, bronchopulmonary dysplasia; DBP, diastolic blood pressure; HFV, high-frequency variability in heart rate; HR, heart rate; LFV, low-frequency variability in heart rate; SBP, systolic blood pressure; $\mathbf{S p O}_{2}$, arterial oxyhemoglobin saturation; SWS, slow-wave sleep 

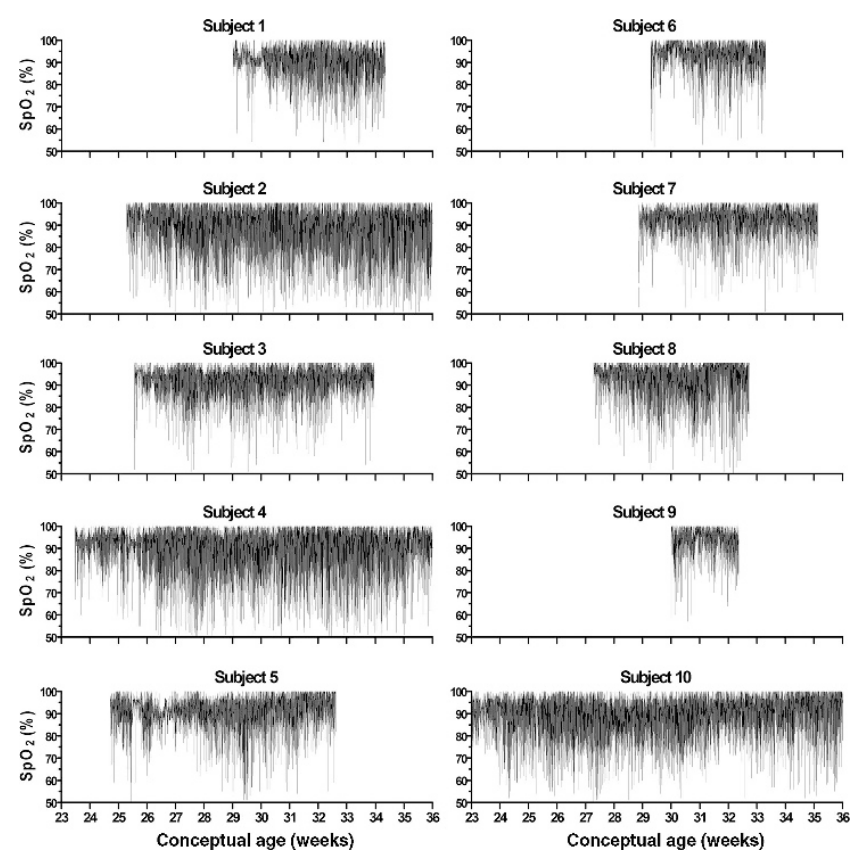

Figure 1. Arterial oxyhemoglobin saturation $\left(\mathrm{SpO}_{2}\right)$ values of BPD infants at 2-min intervals from the start of the neonatal intensive care period.

19 side-motion tests and head-up tilts were performed in SWS until successful tests without signs of arousal were achieved. Polysomnography consisted of continuous monitoring of two electroencephalograms (EEGs) (C3A2, O2A1), one or two electro-oculograms (EOGs), chin and diaphragm muscle surface electromyograms (EMGs), electrocardiograms (ECGs), nasal airflow (RSS100-HR, Hans Rudolph, Kansas City, MI), respiratory movements, $\mathrm{SpO}_{2}$, end-tidal carbon dioxide $\left(\mathrm{EtCO}_{2}\right)($ Capnomac Ultima ULT-SVI, DatexOhmeda, Helsinki, Finland), and BP (Fig. 2). BP was measured by the photoplethysmographic technique (Finapres or Finometer, Finapres Medical Systems, Amsterdam, The Netherlands) with the cuff placed around the infant's wrist (26,27). Data were collected by either the Amlab (Amlab Technology Pty Ltd., Sydney, Australia) or Siesta (Compumedics, Abbotsford, Australia) polygraphic system with 16-bit amplitude resolution and 100to 512-Hz data sampling: ECG and EMG signals were collected using $200 \mathrm{~Hz}$ (Amlab) or $512 \mathrm{~Hz}$ (Siesta), $\mathrm{SpO}_{2}$ at $1 \mathrm{~Hz}$ and other signals using $100 \mathrm{~Hz}$ (Amlab) or $128 \mathrm{~Hz}$ (Siesta). $\mathrm{SpO}_{2}$ data shown in Figure 1 were collected automatically by an intensive care monitoring system (Agilent Technologies and Clinisoft, Anandic Medical Systems, Diessenhofen, Switzerland) at a sampling rate of one sample per 2 min during the whole intensive care treatment period.

In the side-motion test, the infant was first elevated in a horizontal position. After a baseline period of $>30 \mathrm{~s}$, a back-and-forth side motion with a radius of $0.5 \mathrm{~m}$ was performed within $3-5 \mathrm{~s}$. In the tilt test, the infant was tilted manually from the horizontal position to a 45-degree
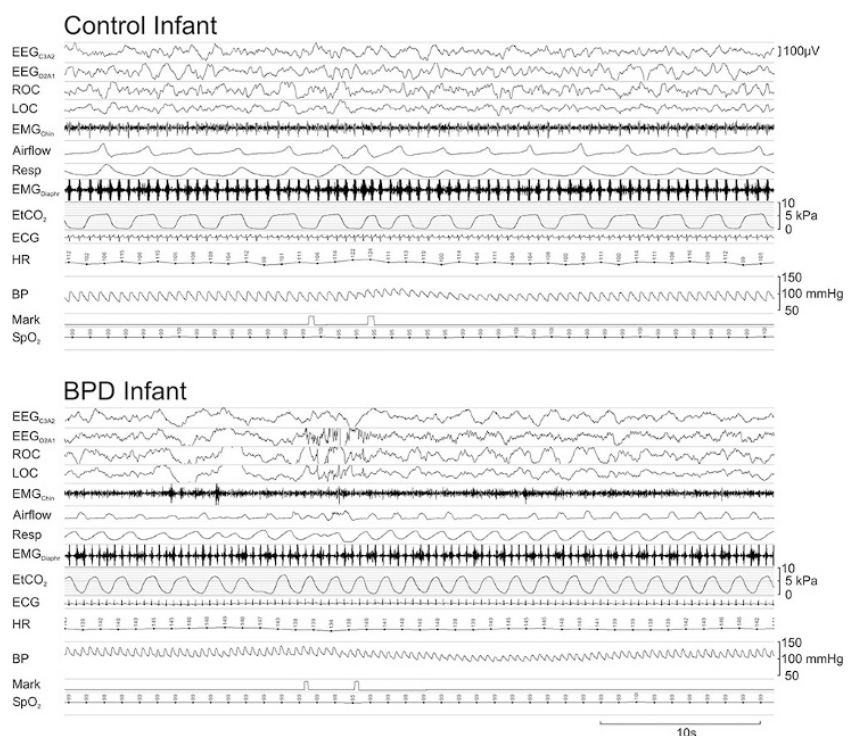

Figure 2. Examples of original polygraphic recording during side-motion tests for a control (upper panel) and a BPD infant (lower panel). The onset and end of the side-motion test are indicated by the mark signal. Resp, respiratory movements; $\mathrm{EtCO}_{2}$ end-tidal carbon dioxide content of the breathing air; $\mathrm{SpO}_{2}$, pulse oximeter arterial oxyhemoglobin saturation. Amplitude of EEG and EOG signals shown with a scale bar.

head-up position in $2-3 \mathrm{~s}$. The tilt position was maintained for $45 \mathrm{~s}$, after which the infant was returned to the horizontal position. The BP measuring cuff was held at heart level throughout the test. After each test, the infant was kept motionless for at least 1 min before another test. Two researchers closely observed the infant for any signs of arousal, such as facial, finger, or hand movements.

Data and statistical analysis. Data were converted into the European Data Format and further analyzed by use of Somnologica sleep polygraphy software (MedCare, Reykjavik, Iceland), together with special purpose software. Sleep staging was performed visually by the researcher using the criteria defined by Guilleminault and Souquet (28). ECG R-wave and BP systolic and diastolic value detection was done under visual verification from the raw data with the use of special purpose software. All signals were analyzed at the accuracy of the original sampling rate with care to exclude tests with any evidence of arousal, including movements of the face, body, or extremities, or a clear change in EEG frequency beyond the time of the movement artifact caused by the test. It was evident from the preliminary tests that any such arousal event would significantly alter the test results (22).

One spontaneous arousal from SWS was selected, and the slope of change in HR (beats/min/min) during the first $10 \mathrm{~s}$ from onset of that arousal was calculated. HR variability analysis was determined from 2-min segments of steady SWS. The HR variability analyzer used oversampling techniques to

Table 1. Demographic data for infants with BPD

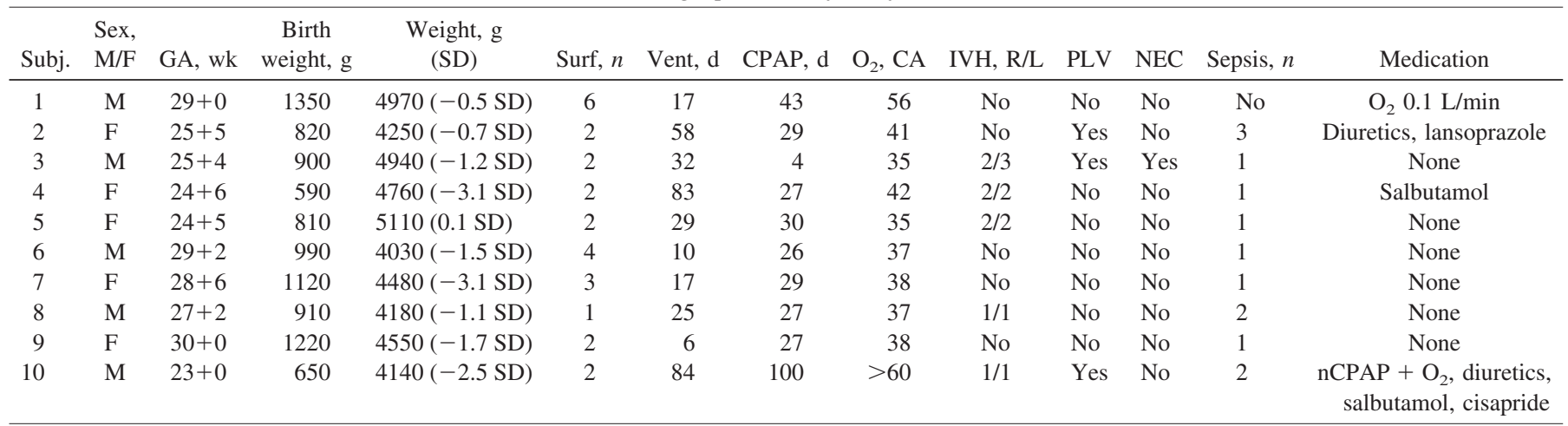

Weight and height are presented in respect to normal values (25), and deviation from the mean of the normal population is in parentheses as the $z$ score. GA, gestational age; IVH, intraventricular hemorrhage; L, left; nCPAP, nasal continuous positive airway pressure; NEC, necrotizing enterocolitis; PLV, periventricular leukomalacia; R, right; Surf, surfactant (Curosurf, Chiesi, Parma, Italy); Vent, ventilatory support; $\mathrm{O}_{2}$, oxygen supply. 
interpolate up to 10 times the original $200-$ or $512-\mathrm{Hz}$ sampling rate. HR variability spectra were calculated from these HR data and integrated over three frequency bands: $0.04-0.15$ (low-frequency variability, LFV), $0.15-1.0$ $\mathrm{Hz}$ (high-frequency variability, HFV), and total power (TP) $0.003-1.0 \mathrm{~Hz}$. The HR variability indices were logarithmically transformed $\left(\log _{10}\right)$ to normalize the distribution. For the statistical analysis of side-motion and tilt tests, four time epochs were included: $20-5 \mathrm{~s}$ before the test, $0-5 \mathrm{~s}$ and $10-15 \mathrm{~s}$ from the test onset, and 25-40 s from the test onset or the end of the tilt. Two-way, repeated-measures analysis of variance (ANOVA) (repeatedmeasures general linear model) were calculated using SPSS 12.0.1 software (SPSS Inc., Chicago, IL). Tukey's (group effects) or Sidak's (within-subject effects) pairwise multiple-comparison test was performed when the ANOVA showed a significant difference. The results are presented in the form mean \pm standard (SD) (range).

\section{RESULTS}

The mean polygraphic recording time was $3.3 \pm 1.6 \mathrm{~h}$ and average total sleep time $2.6 \pm 1.3 \mathrm{~h}$. Sleep stage distribution was $30 \%$ of light NREM, $46 \%$ of SWS, and $25 \%$ of rapid eye movement (REM) sleep in controls and, respectively, 26\%, $49 \%$, and $26 \%$ in BPD infants $(p=0.42)$. Six controls and five BPD infants had obstructive events during sleep with mean obstructive apnea/hypopnea indices of $0.1 \pm 0.3$ $(0-1.4) / \mathrm{h}$ and $0.9 \pm 1.0(0-2.5) / \mathrm{h}(p=0.008)$. The percentage of arousals in response to tests was similar between the groups. The side-motion and tilt tests were difficult to perform without any signs of arousal in some controls and BPD infants, and thus the number of tests performed varied greatly. On average, successful side-motion tests were achieved after 6.0 tests in the controls and 6.3 tests in the BPD infants $(p=0.86)$, and after 4.4 and 3.3 tilt tests, respectively ( $p=0.29)$.

Side-motion test. Figure 2 presents a typical reaction of a control infant to side motion and an example of decrease in reaction of $\mathrm{BP}$ in one of the $\mathrm{BPD}$ infants. Controls consistently showed a biphasic reaction of both $\mathrm{HR}$ and $\mathrm{BP}$ with an initial increase $(p<0.0001)$ followed by decrease [HR, not significant (ns), systolic BP (SBP), $p=0.002$, diastolic BP (DBP), $p=0.02$ ] and normalization (Fig. 3). Half the BPD infants showed clearly altered BP responses to side motion compared with controls, with a mild or absent initial BP response followed by a marked decrease in BP (Fig. 4). Three BPD infants showed a decrease in HR at start of the side motion, and one of the BPD infants (subject 2) had a flat HR and BP response. Although the mean values did not differ significantly between groups, the intersubject variability of HR
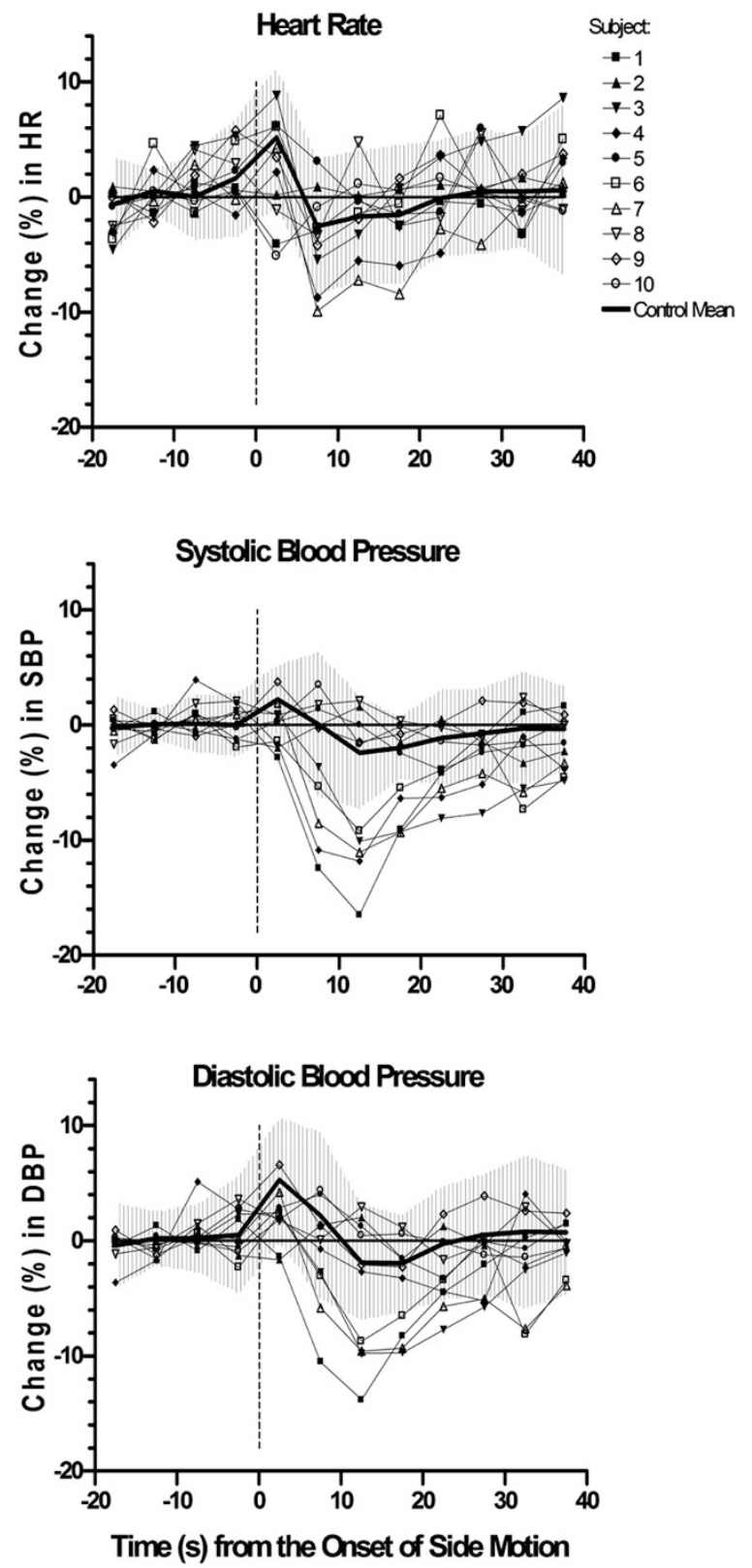

Figure 4. HR and BP responses to linear side motion in BPD and control infants. For the control infants, a mean value $\pm 2 \mathrm{SD}$ area is presented. Vertical line indicates onset of side motion. Values are percentages of changes in comparison with premotion period.
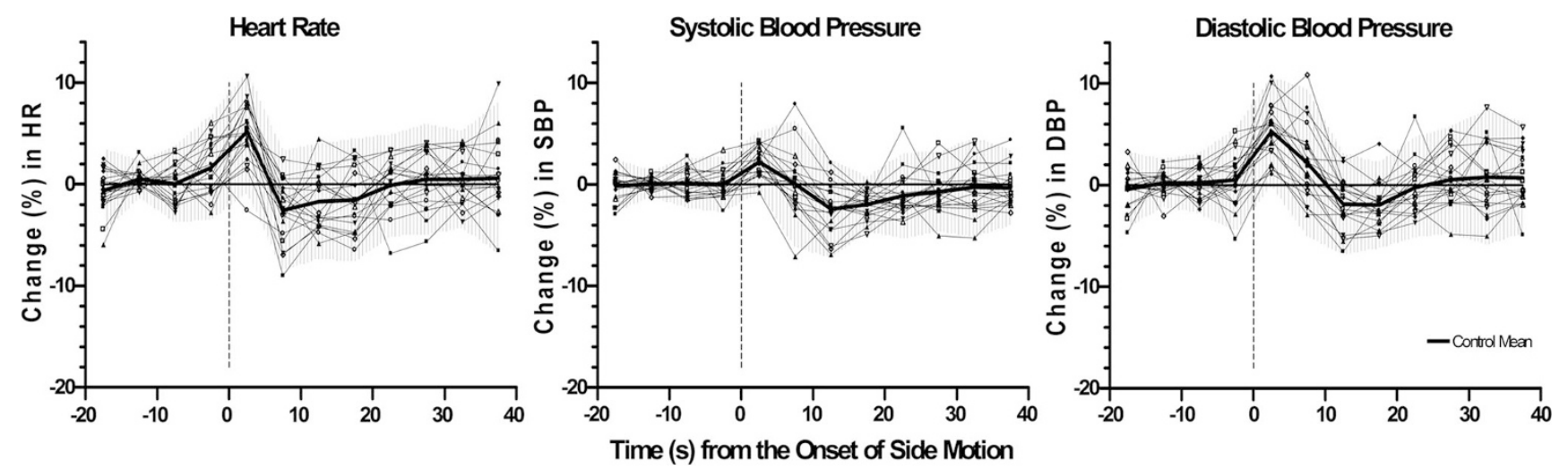

Figure 3. HR and BP responses to linear side motion in control infants. A mean value with \pm 2 SD area is also presented. Vertical line indicates the onset of side motion. Values are the percentages of changes in comparison with premotion period. 
(control mean: $p=0.04$; group mean: $p=0.13$, ns), SBP (control mean: $p<0.0001$; group mean $p<0.0001$ ), and DBP (control mean: $p<0.005$; group mean: $p<0.0001$ ) responses from the control and group mean response were significantly higher in BPD infants compared with those in controls. The degree of response deviation from the control or group mean at 10-15 s from the test onset correlated with none of the following variables: gestational age; estimated amount of total hypoxic exposure (area under $\mathrm{SpO}_{2}$ of $100 \%$ or $85 \%$ ); number of $\mathrm{SpO}_{2}$ desaturations $<85 \%$ during the early neonatal period (Fig. 1); time spent on the ventilator; CPAP support or supplementary oxygen; or the presence of IVH, PLV, NEC, or sepsis during the neonatal period; or drugs used (Table 1). However, successful tests without evidence of an arousal were obtained with fewer tests in BPD infants with suppressive BP responses (subjects $1,3,4,6,7)(4.2$ tests on average) than in the remaining BPD infants (8.4 tests on average) $(p=0.04)$; but in both groups, the number of tests did not deviate significantly from those in normal controls.

Head-up tilting. Controls showed marked intersubject variation in HR and BP responses to head-up tilt. On average, the controls presented with an initial increase, followed by a modest but sustained decrease in HR and BP (Fig. 5). BPD infants presented with a greater intersubject variability in SBP (control mean: $p<0.0001$; group mean $p=0.0001$ ) and DBP (control mean: $p<0.005$; group mean: $p=0.01$ ) responses from the control and group mean than did controls, whereas the mean values did not differ significantly from each other (Fig. 6). Of the BPD infants, four of 10 presented with sustained increases in BP. The degree of response deviation from the control or group mean at 10-15 s from tilt onset showed no correlation with gestational age; estimated amount of total hypoxic exposure; number of $\mathrm{SpO}_{2}$ desaturations $<85 \%$ during the early neonatal period; time spent on a ventilator, CPAP, or supplementary oxygen; or the presence of IVH, PLV, NEC, sepsis, or drugs used during the neonatal period.

$H R$ responses to arousal and HR variability. During spontaneous arousals from SWS, HR accelerated at $147 \pm 45$ beats $/ \mathrm{min} / \mathrm{min}$ in the controls and at $186 \pm 58$ beats $/ \mathrm{min} / \mathrm{min}$ $(p=0.05)$ in BPD infants. Between the groups, the baseline HR level did not differ.
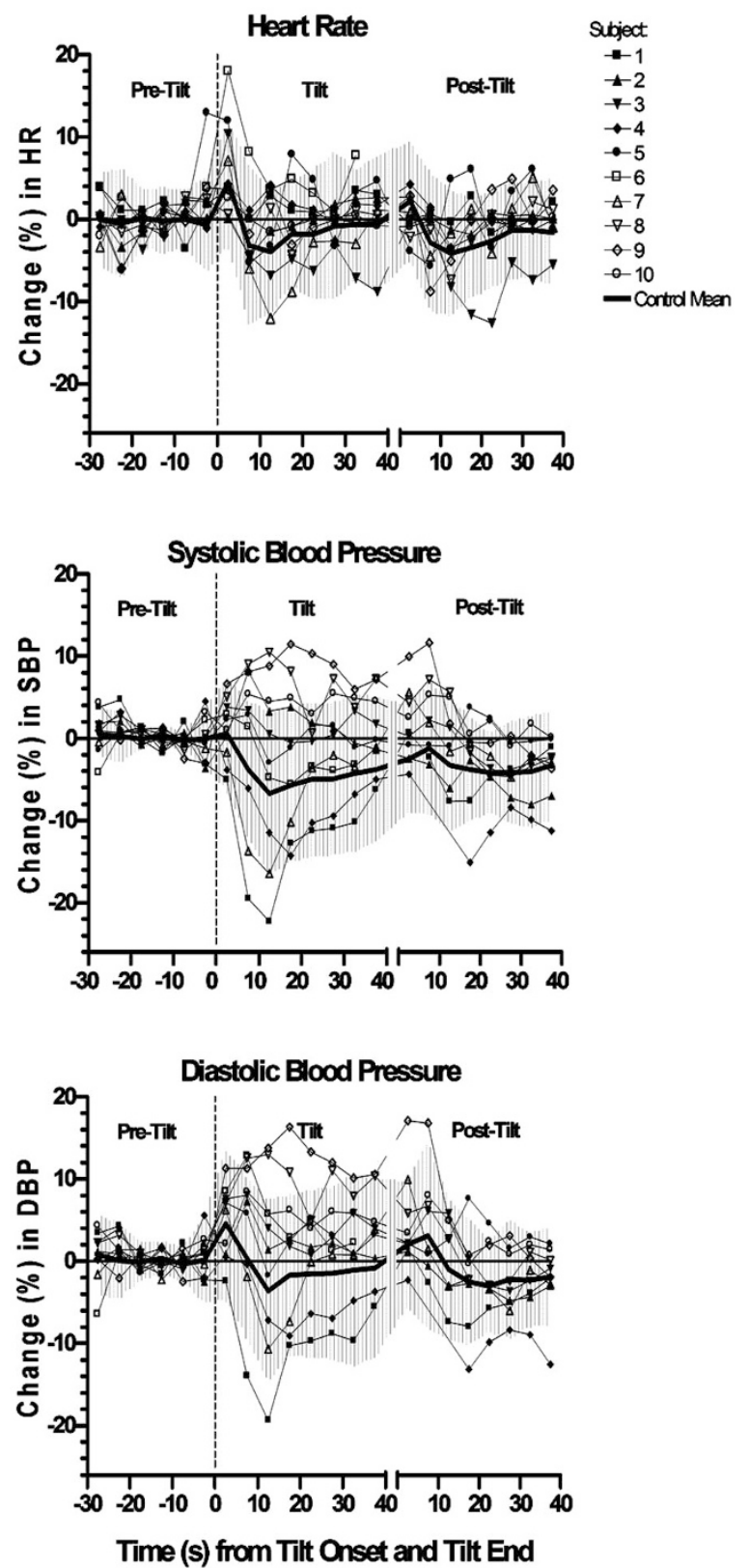

Figure 6. HR and BP responses to 45-degree fast head-up tilt in BPD and control infants. For the control infants, a mean value $\pm 2 \mathrm{SD}$ area is presented. Vertical line indicates onset of tilt test. Values presented as percentages of changes in comparison with pretilt period.
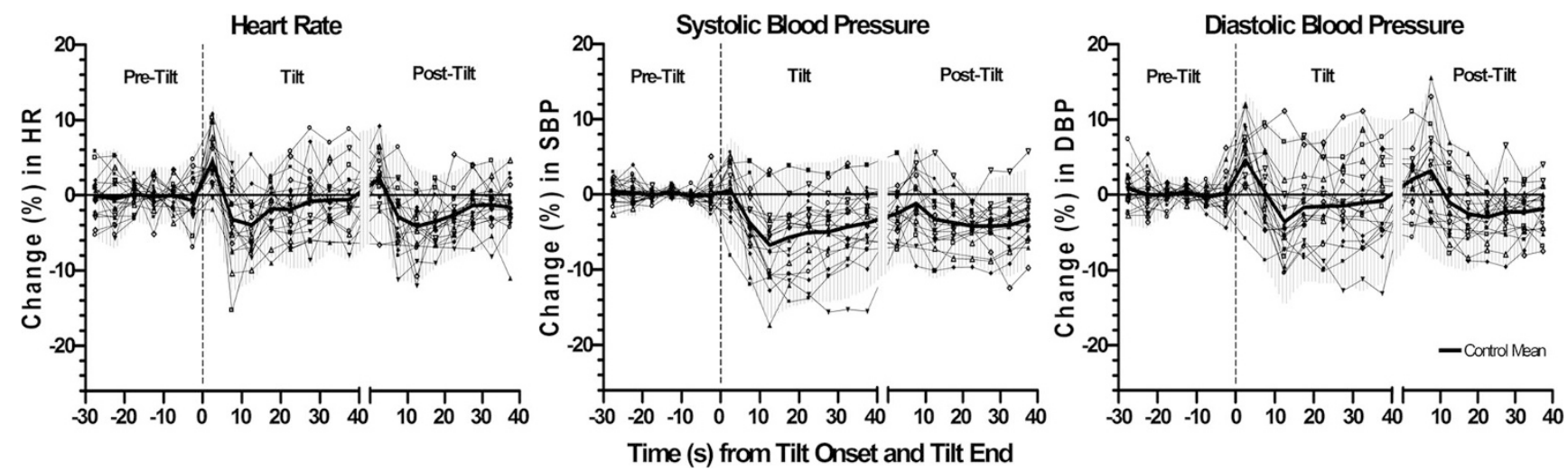

Figure 5. HR and BP responses to 45-degree fast head-up tilt in control infants. A mean value with \pm 2 SD area is also presented. Vertical line indicates onset of tilt test. Values presented as percentages of changes in comparison with pretilt period. 
Table 2. HR variability in SWS

\begin{tabular}{lcccc}
\hline & LFV & HFV & TP & LFV/HFV \\
\hline Control & $2.9 \pm 0.3$ & $2.8 \pm 0.3$ & $3.3 \pm 0.2$ & $1.0 \pm 0.1$ \\
BPD & $3.0 \pm 0.4$ & $3.0 \pm 0.4$ & $3.5 \pm 0.2^{*}$ & $1.0 \pm 0.2$ \\
\hline
\end{tabular}

Values are $\log _{10}$ value, arbitrary units; TP, TP of HR variability. $* p<0.05$.

HR variability results are presented in Table 2. No significant differences between the controls and BPD infants in $\mathrm{LFV}, \mathrm{HFV}$, or LFV/HFV, but TP was modestly increased in BPD infants $(p=0.02)$. Breathing frequencies were similar between the groups during the 2-min HR variability test periods $(p=0.99)$. BPD infants with suppressive BP responses (subjects $1,3,4,6,7$ ) in the side-motion test showed similar increases in HR $(p=0.79)$ and similar HR variability to those of the remaining BPD infants.

\section{DISCUSSION}

We found that a proportion of premature infants with BPD and a history of repetitive short-lasting hypoxic episodes showed altered vestibulo-mediated cardiovascular control at a corrected age of 2-4 mo. This finding was seen as a marked monophasic BP decrease in reaction to side motion in half the BPD infants instead of the biphasic BP reaction in controls, with a short-lasting increase followed by a modest decrease in $\mathrm{BP}$ observed in the latter. Altered vestibulo-mediated cardiovascular control is also suggested by the greater intersubject variability in BP responses to head-up tilt than in controls. None of the BPD infants studied showed responses in both side-motion and tilt tests similar to those of the controls.

Only two BPD infants had HR and BP responses to side motion comparable with those of the controls. One BPD infant (subject 2), with substantial neonatal exposure to intermittent hypoxia later followed by intermittent hypoxic episodes due to severe laryngotracheomalacia, had flat $\mathrm{HR}$ and BP responses similar to those observed in infants with univentricular heart and sustained hypoxia (22).

In tilt tests, control infants showed a biphasic response to head-up tilt with an initial increase in HR followed by a sustained decrease in SBP. BPD infants presented with greater intersubject variability in BP responses to head-up tilt than controls. Vestibular dysfunction is expected to result in greater variability in BP during postural changes (10). Therefore, the increased variability in the present study likely results from the dysfunction of vestibulo-mediated cardiovascular control observed, instead of altered baroreflex function. Of the BPD infants, four of 10 presented with a sustained increase in BP during tilt. Similar responses with an increase in BP have been observed in ALTE infants $(11,12)$, in infants with univentricular heart (22), and in cats after bilateral vestibular lesions (10).

The current study revealed no specific risk factor or reason for the altered cardiovascular control in BPD infants that would separate the BPD infants with normal from those with altered side-motion and tilt-test results. In particular, the deviation in $\mathrm{HR}$ and $\mathrm{BP}$ reactions from the average control or BPD infant reaction did not correlate with the extent of hypoxic challenge. Nevertheless, individual vulnerability to hypoxia may be more important in the development of cardiovascular control than is the absolute extent of hypoxic exposure. It is generally assumed that most of the negative effects of prematurity on brain function result from hypoxia (29).

Another potential cause of this vestibular dysfunction is exposure to gentamicin (10 of 10 BPD infants) with known vestibulotoxicity $(30,31)$ or vancomycin (nine of $10 \mathrm{BPD}$ infants) with known, but rare, ototoxicity (32). All BPD infants received gentamicin after birth or during sepsis periods, and all but one received vancomycin as well (Table 1). Hence, gentamicin- or vancomycin-induced vestibular damage is possible even though (1) gentamicin and vancomycin serum levels were checked and drug dose was adjusted if necessary, (2) all the BPD infants passed auditory brainstem response tests (33), and (3) neonates are assumed to be more resistant to vestibulotoxic effects of aminoglycosides than are older children and adults $(34,35)$. No differences appeared in gentamicin or vancomycin use or in their serum concentrations between BPD infants with a normal or an altered sidemotion response. Altered response to side motion also appeared in one BPD infant (subject 7), with only a short and low serum level exposure to gentamicin and no vancomycin exposure (Table 1).

One possible reason for the difference observed between the groups may be prematurity itself or the difference in postnatal age. However, no age-related differences existed within the groups in side-motion or tilt test results. Furthermore, in very premature infants, baroreceptor-related cardiovascular control is already intact after birth before 26 wk of gestation along with gradually improving cardiac function (36).

Despite the altered cardiovascular responses to side motion and tilt, BPD infants showed HR variability similar to that of controls and even increased HR responsiveness to arousal. Traditional cardiovascular studies would therefore have been unlikely to reveal the altered cardiovascular control in these infants. We suggest that, in very low birth weight infants, altered control may contribute to the increased risk of SIDS (16).

In conclusion, we show that a significant proportion of premature infants with chronic lung disease have abnormal function of vestibulo-mediated cardiovascular control at a corrected age of 2-4 mo.

\section{REFERENCES}

1. Harper RM 2000 Sudden infant death syndrome: a failure of compensatory cerebellar mechanisms? Pediatr Res 48:140-142

2. Doba N, Reis DJ 1974 Role of the cerebellum and the vestibular apparatus in regulation of orthostatic reflexes in the cat. Circ Res 40:9-18

3. Lutherer LO, Lutherer BC, Dormer KJ, Janssen HF, Barnes CD 1983 Bilateral lesions of the fastigial nucleus prevent the recovery of blood pressure following hypotension induced by hemorrhage or administration of endotoxin. Brain Res 269:251-257

4. Waters KA, Meehan B, Huang JQ, Gravel RA, Michaud J, Cote A 1999 Neuronal apoptosis in sudden infant death syndrome. Pediatr Res 45:166-172

5. Kinney HC, Filiano JJ, White WF 2001 Medullary serotonergic network deficiency in the sudden infant death syndrome: review of a 15 -year study of a single dataset. J Neuropathol Exp Neurol 60:228-247

6. Kinney HC, McHugh T, Miller K, Belliveau RA, Assmann SF 2002 Subtle developmental abnormalities in the inferior olive: an indicator of prenatal brainstem injury in the sudden infant death syndrome. J Neuropathol Exp Neurol 61:427-441 
7. Diagne M, Delfini C, Angaut P, Buisseret P, Buisseret-Delmas C 2001 Fastigiovestibular projections in the rat: retrograde tracing coupled with gammaamino-butyric acid and glutamate immunohistochemistry. Neurosci Lett 308:49-53

8. Persson PB 1996 Modulation of cardiovascular control mechanisms and their interaction. Physiol Rev 76:193-244

9. Timmers HJ, Wieling W, Karemaker JM, Lenders JW 2003 Denervation of carotid baro- and chemoreceptors in humans. J Physiol 553:3-11

10. Jian BJ, Cotter LA, Emanuel BA, Cass SP, Yates BJ 1999 Effects of bilateral vestibular lesions on orthostatic tolerance in awake cats. J Appl Physiol 86:15521560

11. Fox GP, Matthews TG 1989 Autonomic dysfunction at different ambient temperatures in infants at risk of sudden infant death syndrome. Lancet 2:1065-1067

12. Harrington C, Kirjavainen T, Teng A, Sullivan CE 2002 Altered autonomic function and reduced arousability in apparent life-threatening event infants with obstructive sleep apnea. Am J Respir Crit Care Med 165:1048-1054

13. Jones KL, Krous HF, Nadeau J, Blackbourne B, Zielke HR, Gozal D 2003 Vascular endothelial growth factor in the cerebrospinal fluid of infants who died of sudden infant death syndrome: evidence for antecedent hypoxia. Pediatrics 111:358-363

14. Smith GC, Wood AM, Pell JP, White IR, Crossley JA, Dobbie R 2004 Secondtrimester maternal serum levels of alpha-fetoprotein and the subsequent risk of sudden infant death syndrome. N Engl J Med 351:978-986

15. Anderson HR, Cook DG 1997 Passive smoking and sudden infant death syndrome: review of the epidemiological evidence. Thorax 52:1003-1009

16. Sowter B, Doyle LW, Morley CJ, Altmann A, Halliday J 1999 Is sudden infant death syndrome still more common in very low birthweight infants in the 1990s? Med J Aust 171:411-413

17. Malloy MH 2004 Sudden infant death syndrome among extremely preterm infants: United States 1997-1999. J Perinatol 24:181-187

18. Kahn A, Groswasser J, Rebuffat E, Sottiaux M, Blum D, Foerster M, Franco P, Bochner A, Alexander M, Bachy A, et al 1992 Sleep and cardiorespiratory characteristics of infant victims of sudden death: a prospective case-control study. Sleep $15: 287-292$

19. Kahn A, Sawaguchi T, Sawaguchi A, Groswasser J, Franco P, Scaillet S, Kelmanson I, Dan B 2002 Sudden infant deaths: from epidemiology to physiology. Forensic Sci Int 130:S8-S20

20. Waters KA, Gonzalez A, Jean C, Morielli A, Brouillette RT 1996 Face-straightdown and face-near-straight-down positions in healthy, prone-sleeping infants. J Pediatr 128:616-625

21. Sullivan FM, Barlow SM 2001 Review of risk factors for sudden infant death syndrome. Paediatr Perinat Epidemiol 15:144-200
22. Kirjavainen T, Viskari S, Pitkanen O, Jokinen E 2005 Infants with univentricular heart have reduced heart rate and blood pressure responses to side motion and altered responses to head-up tilt. J Appl Physiol 98:518-525

23. Oyen N, Markestad T, Skaerven R, Irgens LM, Helweg-Larsen K, Alm B, Norvenius G, Wennergren G 1997 Combined effects of sleeping position and prenatal risk factors in sudden infant death syndrome: the Nordic Epidemiological SIDS Study. Pediatrics 100:613-621

24. Jobe AH, Bancalari E 2001 Bronchopulmonary dysplasia. Am J Respir Crit Care Med 163:1723-1729

25. Haschke F, van't Hof MA 2000 Euro-Growth references for length, weight, and body circumferences. Euro-Growth Study Group. J Pediatr Gastroenterol Nutr 31:S14-S38

26. Drouin E, Gournay V, Calamel J, Mouzard A, Roze JC 1997 Feasibility of using finger arterial pressure in neonates. Arch Dis Child Fetal Neonatal Ed 77:F139-F140

27. Harrington C, Kirjavainen T, Teng A, Sullivan CE 2001 Cardiovascular responses to three simple, provocative tests of autonomic activity in sleeping infants. J Appl Physiol 91:561-568

28. Guilleminault C, Souquet M 1982 Appendix II: Scoring criteria. In: Guilleminault C (ed) Sleeping and Waking Disorders: Indications and Techniques. Addison-Wesley Publishing Co., Menlo Park, pp 415-426.

29. Rees S, Inder T 2005 Fetal and neonatal origins of altered brain development. Early Hum Dev 81:753-761

30. Black FO, Pesznecker S, Stallings V 2004 Permanent gentamicin vestibulotoxicity. Otol Neurotol 25:559-569

31. Saunders JE 2005 Vestibulotoxicity: a risk of home aminoglycoside therapy. J Okla State Med Assoc 98:596-600

32. de Hoog M, Mouton JW, van den Anker JN 2004 Vancomycin: pharmacokinetics and administration regimens in neonates. Clin Pharmacokinet 43:417440

33. Sininger YS, Cone-Wesson B, Folsom RC, Gorga MP, Vohr BR, Widen JE, Ekelid M, Norton SJ 2000 Identification of neonatal hearing impairment: auditory brain stem responses in the perinatal period. Ear Hear 21:383-399

34. Aust G 2001 Vestibulotoxicity and ototoxicity of gentamicin in newborns at risk. In Tinnitus J 7:27-29

35. de Hoog M, van Zanten BA, Hop WC, Overbosch E, Weisglas-Kuperus N, van den Anker JN 2003 Newborn hearing screening: tobramycin and vancomycin are not risk factors for hearing loss. J Pediatr 142:41-46

36. Shekhawat PS, Sasidharan P, Lewis DA 2001 Myocardial performance and baroreceptor reflexes in preterm neonates: an echocardiographic evaluation using the tilt-table test. Pediatr Cardiol 22:465-470 